\title{
Visual search and foraging compared in a large-scale search task
}

\author{
Alastair D. Smith · Bruce M. Hood • \\ Iain D. Gilchrist
}

Received: 12 April 2007/Revised: 13 November 2007 / Accepted: 11 December 2007/Published online: 10 January 2008

(C) Marta Olivetti Belardinelli and Springer-Verlag 2008

\begin{abstract}
It has been argued that visual search is a valid model for human foraging. However, the two tasks differ greatly in terms of the coding of space and the effort required to search. Here we describe a direct comparison between visually guided searches (as studied in visual search tasks) and foraging that is not based upon a visually distinct target, within the same context. The experiment was conducted in a novel apparatus, where search locations were indicated by an array of lights embedded in the floor. In visually guided conditions participants searched for a target defined by the presence of a feature (red target amongst green distractors) or the absence of a feature (green target amongst red and green distractors). Despite the expanded search scale and the different response requirements, these conditions followed the pattern found in conventional visual search paradigms: feature-present search latencies were not linearly related to display size, whereas feature-absent searches were longer as the number of distractors increased. In a non-visually guided foraging condition, participants searched for a target that was only visible once the switch was activated. This resulted in far longer latencies that rose markedly with display size. Compared to eye-movements in previous visual search studies, there were few revisit errors to previously inspected locations in this condition. This demonstrates the important distinction between visually guided and nonvisually guided foraging processes, and shows that the visual search paradigm is an equivocal model for general search in any context. We suggest a comprehensive model of human spatial search behaviour needs to include search
\end{abstract}

A. D. Smith $(\bowtie)$ B. M. Hood · I. D. Gilchrist

Department of Experimental Psychology, University of Bristol, 12 a Priory Road, Bristol BS8 1TU, UK

e-mail: Alastair.Smith@bristol.ac.uk; alastair.smith@bris.ac.uk at a small and large scale as well as visually guided and non-visually guided search.

\section{Introduction}

Searching the world around us is an important everyday human behaviour, whether we are seeking a face in a crowd or trying to locate a lost pair of spectacles. For over thirty years psychologists have been examining the mechanisms that enable such exploratory activity by utilising the visual search paradigm. The standard requirement of a visual search task is for participants to detect the presence (or absence) of a target item, presented on a computer monitor, within an array of distractor items. By manipulating factors such as the number of distractors, and the visual similarity between them and the target, scientists have produced influential models of human search behaviour (e.g. Duncan and Humphreys 1989; Treisman and Gelade 1980). More recently it has been argued that the visual search task represents a well-specified context with which to study more general foraging behaviour (Wolfe 1994), and that some mechanisms identified in visual search tasks are central to foraging. For example, the tendency not to refixate a previously inspected location (Inhibition of Return) has been branded a 'foraging facilitator' (Klein and MacInnes 1999; cf. Hooge et al. 2005).

Despite the putative similarities between visual search and large-scale foraging, however, the two contexts are very different. First, participants in a visual search task are presented with an objective view of a small-scale space, in the vertical plane, which they are themselves seated before. In comparison, foraging requires the individual to be situated within the search space, necessarily requiring 
additional egocentric coding of spatial relations as one moves through the array. Second, the effort required to move from one item to the next is much greater in a largescale context, which necessitates full body movement. And third, foraging often occurs when no visual cue is available to indicate the exact location of the target (i.e. when the lost glasses are hidden under a book). As such, it is unclear whether the visual search task is a good model for foraging behaviour. This was addressed by Gilchrist et al. (2001) who presented participants with a large-scale threedimensional analogue of a visual search task. Participants were situated within an array of film canisters and required to detect the presence or absence of a hidden target (a marble) by visiting each canister, picking it up, and shaking it. As in traditional serial visual search tasks, search time increased linearly with display size, and the target-present to target-absent search slope was 1:2. In contrast, however, participants made fewer erroneous revisits to locations they had already searched in a trial than would be expected from a conventional visual search task (e.g. Gilchrist and Harvey 2000). The authors argue that the increased effort required in foraging, or large-scale search, means that participants are more likely to remember the locations of visited stimuli in order to minimise costly revisits. This suggests that differences in scale and search activity can produce different search profiles.

The issue of physical effort in large-scale search was specifically addressed by Smith et al. (2005) in an automated version of this task. Children aged between 5 and 8 years were situated in a square room surrounded by curtains. Embedded in a raised floor was an array of lights and switches-participants searched for a hidden target (a red light) by pressing switches at each potential location (defined by green lights). In one condition children searched with their dominant hand and in another they used their non-dominant hand, which was deemed to engage more physical effort (e.g. Carlier et al. 1993). When searching with their non-dominant hands, children made more revisits to locations they had previously checked than when using their dominant hand, suggesting that increased effort can also be associated with decreased memory for visited locations. Search efficiency (i.e. latency, number of visits) was found to correlate strongly with short-term spatial memory measures, but not with age or general intelligence. Revisit behaviour was also measured by Ruddle and Lessels (2006) in a virtual reality (VR) task, where participants searched for multiple targets hidden in an array of boxes. Movement was varied by condition, with participants searching whilst seated at a monitor, standing stationary whilst wearing a VR headset, and actively walking through the virtual display. Movement was strongly associated with revisit behaviour, and participants made far fewer revisits when walking through the display.
They also benefited less from environmental cues in this condition, suggesting that search efficiency was related to spatial updating processes provided by body movement (e.g. Gopal et al. 1989).

Tasks such as these have provided an insight into the nature of large-scale search, and how that might relate to visual search behaviour, but their demands are not completely equivalent to those of conventional visual search. Most notably, the target location is not visually distinguishable from the distractors. This means that it is not possible to compare large-scale search with efficient or 'parallel' (Treisman and Gelade 1980) visual search behaviour, or to address the visually guided nature of search. Serial inspection of potential target locations in large-scale search (Gilchrist et al. 2001; Ruddle and Lessels 2006; Smith et al. 2005) is more akin to inefficient visual search behaviour, especially in visual search tasks where target complexity necessitates fixation of individual items (e.g. Klein and MacInnes 1999; Gilchrist and Harvey 2000). Yet these tasks also lack a visually guided search component, other than an indication of possible locations. Perhaps more fundamentally, explicit comparisons between visual search and large-scale search have not taken place in the same context or the same experiment. Ruddle and Lessels (2006) compared different groups on a monitor-based and full-body search, although they did not focus on visually guided aspects of search, and Gilchrist et al. (2001) compared large-scale search profiles to previously reported visual search data in different tasks.

Our aim in the present study was to directly compare visually guided search and non-visually guided foraging in the same experimental setting. The mode of response was equivalent across conditions, and we employed a withinsubject design. The study took place in the automated search laboratory described by Smith et al. (2005), which enabled presentation of a target amongst distractors, defined by combinations of colour. In visually guided conditions we compared efficient search, based on the presence of a visual feature, with inefficient search, based on the absence of a feature (see Luck and Hilyard 1990). In the non-visually guided condition, potential locations were indicated by an array of identical lights and participants searched for the target by physically inspecting each location. This is closer to the process of foraging as participants were required to move throughout space whilst searching. This paradigm enables a direct comparison of different search behaviours in an identical context, and also allows us to address whether properties of visual search do transpose to large-scale space, as predicted by Wolfe (1994) and Klein and MacInnes (1999). It could also be argued that there is a greater degree of ecological validity in the present paradigm. Not only do participants actively search the space, but the array is also specified in three 
dimensions, extending away from the participant in actual space. This is closer to the demands of the environment naturally encountered by people.

\section{Method \\ Participants}

Twenty-four students from the University of Bristol participated in the experiment. Their mean age was 20 years (range 18-24 years) and the ratio of males to females was 6:18. All participants had normal or corrected-to-normal vision and full physical mobility.

\section{Apparatus}

The large-scale search laboratory was fully described by Smith et al. (2005). Participants were tested in a square room $(4 \times 4 \times 2.5 \mathrm{~m})$ with a raised platform floor. Embedded into the floor of the room were 49 search locations, arranged in a $7 \times 7$ grid. Each location consisted of two lights (one green, one red) and a switch positioned between them. The apparatus was powered and controlled by a personal computer in an adjoining room, which also recorded the location and timing (with ms accuracy) of each button press made by the participant. The testing space was devoid of obvious landmarks: a dark blue curtain was mounted around the walls (also occluding the entrance), and the floor was covered with featureless grey carpeting. This environment is illustrated in Fig. 1. Participants were monitored by a discretely mounted (i.e. unnoticeable within the laboratory) video camera.

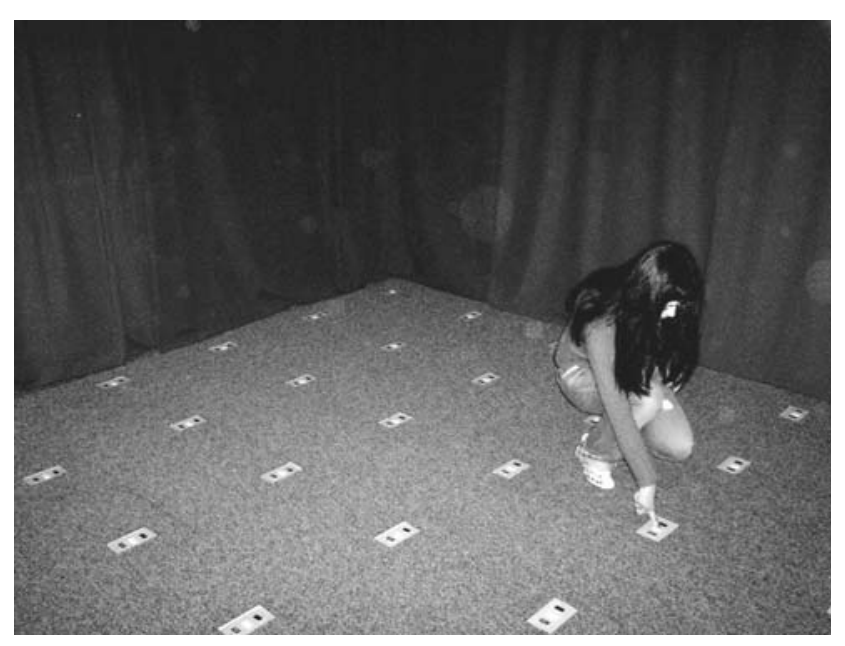

Fig. 1 A participant inspects a potential target location in the Foraging condition by depressing the switch
Design and procedure

Participants performed three blocks of trials in which they searched for a target within a randomised array of distractors. In the Feature-present condition the target was defined by both the red and green lights being lit. On each trial it was displayed amongst an array of distractors, which were defined by only the green light being lit. When participants had located the target, they walked to its location and pressed the switch. In the Feature-absent condition the target was defined by only the green light being lit. It was displayed amongst distractors that were defined by both the red and green lights being lit. Participants again responded once they had located the target by pressing the switch at the respective location. In the Foraging condition all possible target locations were defined by only the green light being lit. As the target location was not distinguishable participants were required to visit each location (pressing the switch) until they located the hidden target. The target was here defined by the red light being illuminated upon depression of the switch.

Each block contained a total of 28 trials, comprising 7 trials of each set size: 5, 10, 15, 20 (locations lit). A target was present in every trial and there were no predictable cues as to where it would appear, although its location was constrained such that each row and column of the grid was utilised. Participants started each trial from the same fixed location: the middle point of a perimeter row, with their back to the wall. Once the target had been located in a trial, all of the lights were extinguished. The next trial was begun when the participant had returned to the starting position. Participants were asked to hold a tennis ball in the hand that would not be used to press the switches to ensure that they only activated one location at a time (in the Foraging task).

\section{Results}

Search time

Figure 1 illustrates overall search times, from the beginning of the trial to the activation of the switch at the target location. Because of the difference between their respective task demands, visual search blocks were analysed separately to the foraging block. A 2 (condition: featurepresent, feature-absent) $\times 4$ (display size: $5,10,15,20$ ) repeated measures Analysis of Variance on mean search times (for the visual search tasks) produced a main effect of condition $(F[1,23]=89.4, P<0.001)$, with significantly slower search times in the feature-absent condition. There was a main effect of display size $(F[3,69]=27.4$, $P<0.001$ ), and a significant interaction between the two 
factors $(F[3,69]=22.7, P<0.001)$ : feature-absent search times rose consistently with larger display sizes, but there was no linear relationship in the feature-present condition. In order to characterise search behaviour in individual tasks the visual search conditions were also analysed separately. In the Feature-present condition there was a main effect of display size upon search time, $F[1,23]=15.4, P<0.001$. However, there was no systematic relationship between the two variables as, for example, search times at display size 10 (mean $=3,350$ $\mathrm{ms}, \mathrm{SD}=530 \mathrm{~ms}$ ) were longer than at display size 20 (mean $=3,227 \mathrm{~ms}, \mathrm{SD}=444 \mathrm{~ms}$ ), thus search time was not linearly related to display size. In the Feature-absent condition there was a main effect of display size $(F[3,69]=26.2, P<0.001)$, and search time consistently increased with display size. The slope showed an increase of $94.8 \mathrm{~ms}$ per item, with an intercept of 3,057 ms. In the Foraging condition, there was a main effect of display size $(F[3,69]=100, P<0.000)$, as search time markedly and consistently increased with display size. Here, the search slope showed an increase of $789.6 \mathrm{~ms}$ per item, with an intercept of 4,011 ms (Fig. 2).

\section{Revisit data}

In the Foraging condition, the target location was not visible, and thus there was a possibility that participants could

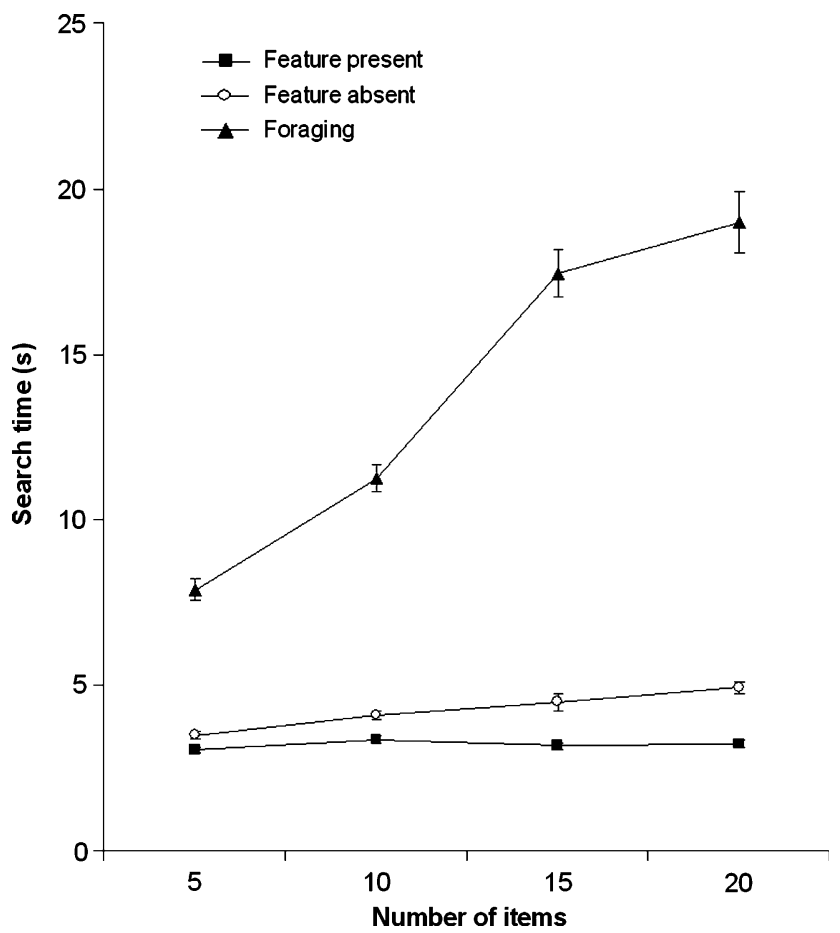

Fig. 2 Mean search time (in seconds) to activate the switch at the target location. Bars illustrate standard error of the mean make erroneous revisits to locations that they had already checked within a single trial. Fourteen participants made no revisit errors at all. For a display size of 5, single revisits (from the remainder of participants) to a checked location made up a mean of $0.17 \%$ of the total visits. For a display size of 10 , a mean of $0.1 \%$ of trials were single revisits, and for a display size of 15 , a mean of $1.6 \%$ of trials were single revisits. When there were 20 possible target locations, a mean of $1.4 \%$ of trials were single revisits, and in one trial a participant made a second revisit to a previously checked location. A single factor (display size: 5, 10, 15, 20) repeated measures Analysis of Variance found no relationship between display size and the number of revisits made by participants, $F(3,69)=1.582$ ns.

\section{Discussion}

Here a novel automated apparatus was used to compare visually guided and non-visually guided search in largescale space. This was the first direct comparison of visual search and foraging-like behaviour within the same experimental context. In the visually guided conditions participants searched for targets that were visually defined by the presence or absence of a visual feature. In conventional visual search tasks (e.g. Luck and Hilyard 1990) search for feature-present targets is efficient and not linearly related to the number of distractors in the array. In the present experiment participants walked to the target location (defined by the presence of an additional red light amongst green distractors) and activated the respective switch. Larger displays were not associated with longer response times: whilst there was a statistical effect of size, there was no linear relationship between the factors. Feature-absent targets usually engender inefficient profiles in visual search experiments: participants tend to serially inspect each item (due to increased visual complexity) and so search times are longer for larger display sizes. Here participants walked to the target location (defined by the absence of a red light amongst red and green distractors) and activated the switch. In this condition search time was linearly related to display size, and search slopes showed an increase of $94.8 \mathrm{~ms}$ per item. This demonstrates that efficient and inefficient search profiles, as measured in traditional visual search tasks presented on a monitor, can also characterise visually guided search in large-scale egocentric space.

In addition to the visually guided conditions, participants were also tested in a non-visually guided search condition where targets were not distinguishable from distractors until they had been visited. This is closer to the process of foraging as participants were required to physically visit and inspect potential locations. Furthermore, foraging can often 
occur when no visual cue is available to distinguish the exact location of the target. All items in the array were defined by green lights, and the target was identified by the illumination of the red light upon activation of the respective switch. Like the inefficient search profiles demonstrated in the feature-absent condition, search time was linearly related to display size, as participants serially inspected each location. Full body movements during search also incurred a greater cost of $790 \mathrm{~ms}$ per additional item. In the foraging condition locations did not alter appearance after inspection (unless it was the target location) and so it was possible for participants to make revisit errors. However, these errors were comparatively small in number (14 participants made no errors at all), and there was no relationship between the display size and the number of revisits made by the remaining participants. This is in line with the findings of Gilchrist et al. (2001) and Ruddle and Lessels (2006), who both report fewer revisit errors during large-scale search tasks.

These findings suggest an equivocal role for the visual search paradigm in modelling and predicting large-scale search behaviour, and more general foraging (e.g. Wolfe 1994). In the visually guided conditions (feature-present, feature-absent) here, search slopes were qualitatively comparable to those reported in conventional computerbased visual search paradigms. This is particularly interesting as the array was displayed in large-scale space and required participants to make full body movements to the target location. This demonstrates that visually guided search can be comparable across spatial scales, plane of presentation (i.e. vertical on monitor-based tasks and horizontal in the present experiment), and response requirements. In comparison, non-visually guided foraging is qualitatively different to that which is measured in visual search paradigms. Whilst the serial nature of inspection is comparable to inefficient visual search, where participants must visually fixate each item in turn, there are distinct differences that derive from the context requirements. First, large-scale body movements take longer to execute than saccades, and so search time is greater affected by additional items in the display. Second, revisits to locations inspected previously in a trial are much less than those usually observed in eye-movement studies of refixations in visual search (Gilchrist and Harvey 2000). The basis of this superior memory for inspected locations in large-scale search may be due to the increased effort required to actively search space, resulting in increased memory deployment for route in order to avoid costly rechecking (Gilchrist et al. 2001). However, Smith et al. (2005) found that increased effort (searching with the non-dominant hand) was associated with poorer memory for inspected locations. Another possibility is that additional cues from kinaesthetic movement through navigational space, such as path integration (Gopal et al. 1989), provide a richer and more powerful representation of exploratory behaviour. Lastly, some form of Inhibition of Return (as predicted by Klein and MacInnes 1999) could account for the comparative lack of revisit error in large-scale search, although this approach would not itself account for the differences observed between small-scale and large-scale search. It therefore seems that visual search tasks can partially equate large-scale search when the visual cues to target location are of an equivalent nature. However, the great differences between visual search and foraging, in terms of visual guidance, scale, movement, and spatial coding, would suggest that search in these two contexts is not qualitatively equivalent.

A key issue here is the definition of the nature and requirements of foraging itself (for an ethological perspective, see Krebs and Davies 1981). Whilst the environment and demands of the present study were more ecologically valid than visual search tasks traditionally employed, it is far removed from a completely naturalistic setting. Furthermore, issues of motivation and reward are very different when comparing the laboratory to ecologically valid contexts. As such, we need to employ greater specificity when making predictions about naturalistic behaviours. The tasks reported here are best conceived as large-scale searches, with either a visually or non-visually guided component. Use of the term foraging has a theoretical precedent (Klein and MacInnes 1999; Wolfe 1994) and has here been used in reference to that discussion, but we suggest that it is discarded in future debate in favour of specification and identification of the precise behaviours being referred to or studied. Spatial behaviour differs greatly across scales and environments, and the coding, representation, utilisation, and retention of spatial information can take a variety of forms depending on the nature of that environment and the task that one is required to perform (see Burgess et al. 1999; Humphreys and Riddoch 2005). In the current study we see that search behaviour can be equivalent across contexts when the properties of the search array (i.e. feature-present, feature-absent) are similar. In contrast, when the target is not initially visible, or the mode of response is notably different, then search mechanisms do not appear to be equivalent.

It is clear that future studies are required to disentangle these issues if we are to develop a full and comprehensive model of human search behaviour. The present experiment represents a starting point in the development of a new paradigm, and we are beginning to utilise this methodology to address other factors that might affect search behaviour, including probabilistic properties of the display (Smith et al. 2007) and inefficiencies of search associated with developmental disorders of spatial cognition (Smith et al. 2006). It is also important to specifically examine the role 
of memory in large-scale search, as it forms a common theme across accounts of visual search behaviour (e.g. Gilchrist and Harvey 2000; Horowitz and Wolfe 1998; Peterson et al. 2001). Garden et al. (2002) found that concurrent spatial tasks interfered with route learning in a naturalistic environment, implicating a role for short-term spatial memory in navigation that could be more closely tested in the present paradigm. In addition to these avenues of enquiry, there are studies that should be conducted in order to more closely equate the demands of large-scale search tasks with the traditional visual search paradigm. For example, there was no target-absent condition in the present experiment, and it could be useful to compare search behaviour (e.g. revisits) between target-present and target-absent trials. Equally, although there was no linear effect of display size on search time in the feature-present search, there was still a statistically significant effect of size. At this stage, the reason for this is unclear; although it could reflect the fact that fixation (at the beginning of each trial) was not controlled in the same way that it is in monitor-based tasks. As a result, there may have been some general effect of the number of items in the display on the amount of visual information initially extracted by participants. Future studies are warranted to more closely examine visual behaviour in the full-body search task, and apparatus such a mobile eye-tracker will prove particularly useful in comparing search behaviour across the variety of spatial scales that we naturally encounter.

Acknowledgments This work was supported by the BBSRC (Grant No. 7/518013) and the Medical Research Council (Grant No.G0401132). We thank Evangelia Liouta in her capacity as an inadvertent model.

\section{References}

Burgess N, Jeffery KJ, O'Keefe J (1999) The hippocampal and parietal foundations of spatial cognition. Oxford University Press, Oxford
Carlier M, Dumont AM, Beau J (1993) Hand performance of French children on a finger-tapping test in relation to handedness, sex, and age. Percept Mot Skills 76:931-940

Duncan J, Humphreys GW (1989) Visual search and stimulus similarity. Psychol Rev 96:433-458

Garden S, Cornoldi C, Logie RH (2002) Visuo-spatial working memory in navigation. Appl Cogn Psychol 16:35-50

Gilchrist ID, Harvey M (2000) Refixation frequency and memory mechanisms in visual search. Curr Biol 10:1209-1212

Gilchrist ID, North A, Hood B (2001) Is visual search really like foraging? Perception 30:1459-1464

Gopal S, Klatzky RL, Smith TR (1989) NAVIGATOR: apsychologically based model of environmental learning through navigation. J Environ Psychol 9:309-331

Hooge IT, Over EA, van Wezel RJ, Frens MA (2005) Inhibition of return is not a foraging facilitator in saccadic search and free viewing. Vis Res 45:1901-1908

Horowitz TS, Wolfe JM (1998) Visual search has no memory. Nature 394:575-577

Humphreys GW, Riddoch MJ (2005) Attention in action: advances from cognitive neuroscience. Psychology Press, Hove

Klein RM, MacInnes WJ (1999) Inhibition of return is a foraging facilitator in visual search. Psychol Sci 10:346-352

Krebs JR, Davies NB (1981) An introduction to behavioural ecology. Blackwell, Oxford

Luck SJ, Hillyard SA (1990) Electrophysiological evidence for parallel and serial processing during visual search. Percept Psychophys 48:603-617

Peterson MS, Kramer AF, Wang RF, Irwin DE, McCarley JS (2001) Visual search has memory. Psychol Sci 12:287-292

Ruddle RA, Lessels S (2006) For efficient navigational search, humans require full physical movement, but not a rich visual scene. Psychol Sci 17:460-465

Smith AD, Gilchrist ID, Hood BM (2005) Children's search behaviour in large-scale space: Developmental components of exploration. Perception 34:1221-1229

Smith AD, Gilchrist ID, Hood BM, Karmiloff-Smith A (2006) Developmental components of large-scale search: evidence from children and individuals with partial genetic deletions. Cogn Process 7:S93-S94

Smith AD, Hood BM, Gilchrist ID (2007) Mechanisms of probabilistic cueing in large-scale search. Perception 36:1402

Treisman AM, Gelade G (1980) A feature integration theory of attention. Cogn Psychol 12:97-136

Wolfe JM (1994) Guided Search 2.0 - a revised model of visual search. Psychon Bull Rev 1:202-238 